\title{
Early growth response 2 and Egr3 are unique regulators in immune system
}

\author{
SINA TAEFEHSHOKR ${ }^{1,2}$, YASHAR AZARI KEY ${ }^{1,2}$, MANSOUR KHAKPOUR, \\ POURYA DADEBIGHLU ${ }^{2}$, AMIN OVEISI ${ }^{2}$ \\ ${ }^{1}$ Young Researchers and Elite Club, Tabriz Branch, Islamic Azad University, Tabriz, Iran \\ ${ }^{2}$ Veterinary Medicine Faculty, Tabriz Branch, Islamic Azad University, Tabriz, Iran \\ ${ }^{3}$ Department of Pathobiology, Faculty of Veterinary Medicine, Tabriz Branch, Islamic Azad University, Tabriz, Iran
}

\begin{abstract}
The immune system is evolved to defend the body against pathogens and is composed of thousands of complicated and intertwined pathways, which are highly controlled by processes such as transcription and repression of cellular genes. Sometimes the immune system malfunctions and a break down in self-tolerance occurs. This lead to the inability to distinguish between self and non-self and cause attacks on host tissues, a condition also known as autoimmunity, which can result in chronic debilitating diseases. Early growth response genes are family of transcription factors comprising of four members, Egr1, Egr2, Egr3 and Egr4. All of which contain three cyc2-His2 zinc fingers. Initially, Egr2 function was identified in the regulation of peripheral nerve myelination, hindbrain segmentation. Egr3, on the other hand, is highly expressed in muscle spindle development. Egr2 and Egr3 are induced due to the antigen stimulation and this signaling is implemented through the $B$ and $T$ cell receptors in the adaptive immunity. T cell receptor signaling plays a key role in Egr 2 and 3 expressions via their interaction with NFAT molecules. Egr 2 and 3 play a crucial role in regulation of the immune system and their involvement in $B$ and $T$ cell activation, anergy induction and preventing the autoimmune disease has been investigated. The deficiency of these transcription factors has been associated to deficient Cbl-b expression, a resistant to anergy phenotype, and expression of effector and activated $T$ cells.
\end{abstract}

Key words: autoimmune diseases, immune system, early growth response, regulator, anergy.

(Centr Eur J Immunol 2017; 42 (2): 205-209)

\section{Early growth response genes: from structure to function}

Early growth response genes are family of transcription factors comprises four members Egr1(Krox-24/NGFI-A), Egr2(Krox-20), Egr3(NGFI-3/Pilot) and Egr4(NGFI-C) which contain three cyc2-His2 zinc fingers binding to the same cognate GC-rich consensus DNA binding motif of 28-30 amino acids, the Egr response element (Fig. 1) [1, $2]$. They are expressed in many different cell types and their expression is rapidly induced in response to mitogens, differentiation, apoptotic signals and tissue injury [3].

Egr1 plays a role in thymocyte development and promotes positive selection of both CD4 and CD8 single positive cells without having a major effect in negative selection, by enhancing expression of anti-apoptosis molecule bcl-2 and Id3 inhibitor [4]. Id3 promotes thymocyte maturation in which its expression is up-regulated in response to TCR signaling during positive selection [5, 6]. Egr1 expression is essential for macrophage lineage differentiation [7].
Egr2 expression is associated with the onset of myelination in the peripheral nervous system and hindbrain development [2]. Egr3 has a vital role in muscle-spindle development [8].

Egr4 that was firstly identified in the central nervous system, is mostly expressed at high levels in the forebrain and has critical role in spermatogenesis in terms of male murine fertility [9]. It is identified as a novel regulator of posterior hindbrain development [10].

\section{Indispensible role of Egr2 and Egr3 in $T$ and $B$ cell development and activation}

Egr2 and Egr3 are expressed in the selected stages of $\mathrm{B}$ and $\mathrm{T}$ cell development in the thymus and bone marrow respectively [11]. They are repressed in immature single positive (ISP) thymocytes and pro-B cells which suggest an indispensible role of Egr molecules for the development of double negative (DN) cells following $\beta$ selection. Double negative cells are lymphocytes bearing a and $\beta$

Correspondence: Sina Taefehshokr, Veterinary Medicine Faculty, Tabriz Branch, Islamic Azad University, Tabriz, Iran, C3, Azin Building, Kouhestan Street, Roshdiye Town 5155933795 Tabriz, Iran, e-mail: sinataefehshokr@gmail.com Submitted: 4.05.2016; Accepted: 28.06.2016 


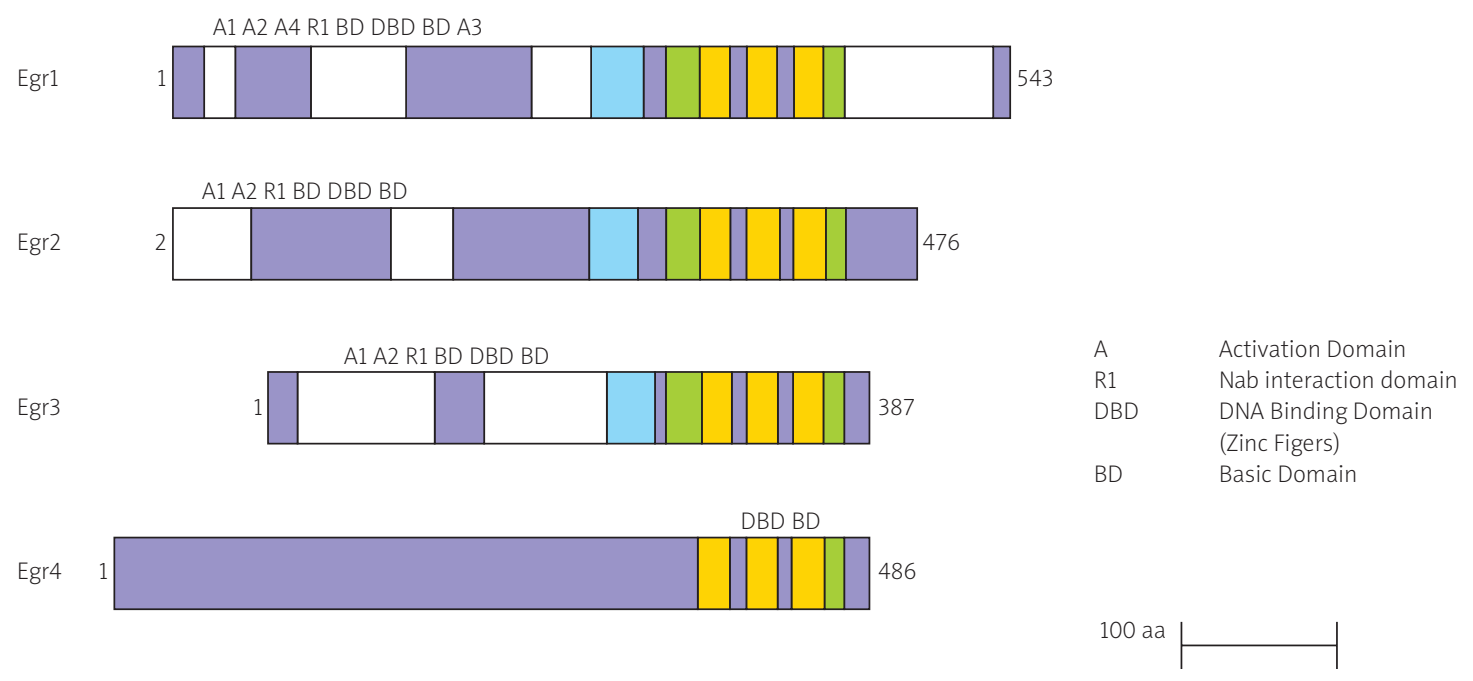

Fig. 1. Schematic representing structure of four members of the Egr family [1]

T cell receptor but lacking CD4 and CD8 markers [12]. Egr2 expression is withdrawn after $\beta$ selection for the differentiation of immature single positive (ISP) cells to double positive (DP) cells [11]. Double positive cells express both CD4 and CD8 cell surface markers during T cell development [12]. T and B cells from Egr 2 and 3 knock out showed severe defects in proliferation and IL-2 production after antigenic receptor stimulation in vitro [13].

\section{Egr2 is vital regulator in a growth- suppressive manner}

Early studies highlighted the role of Egr genes in cell proliferation [14]. Egr2 has been suggested as a mediator of the PTEN growth-suppressive signaling pathway [15]. In this process PTEN, a gene located in the cytoplasm acting as a tumor suppressor, induces Egr2 expression and then Egr2 directly transactivates expression of two pro-apoptotic proteins of Bcl-2 family, BNIP3L and BAK, localized at mitochondria and alter the permeability of mitochondrial membranes, releasing cytochrome $\mathrm{c}$ and activating caspase 3,8 and 9 [16]. This suggested that exogenous expression of Egr2 can have growth suppressive effects and Egr2 may be an excellent target molecular gene therapy to treat a variety of cancers. A recent study also shows that Egr2 is growth inhibitory when overexpressed in tumor cells and induced CEBPB, transcription factor that contributes to OIS in primary human and mouse fibroblasts that express oncogenic Ras or BRAF [17].

In addition, Egr2 attenuates pre-osteoclast proliferation by restraining cell-cycle progression in which Egr2 silencing can result in accelerated proliferation likely due to the increased cFms expression and signalling [18].
Constitutive expression of Egr2 in schwann cells reveals a major function of Egr2 in which it suppresses the c-Jun NH2-terminal protein kinase (JNK)-c-Jun pathway, activation of which is required for both proliferation and death. Thus, Egr2 can coordinately control suppression of mitogenic and death responses [19]. So, this dual function allows Egr2 to suppress both cell division and death through a single action namely inactivation of the JNK-c-Jun pathway. In contrast, Egr2 knockdown inhibit proliferation and spheroidal growth in vitro and induced regression of Ewing sarcoma xenografts, reduced cell cycle progression through $\mathrm{S}$ phase and reduced cell viability [20].

\section{Maintenance of Peripheral Tolerance Mechanisms}

Anergy, apoptosis and the action of regulatory T cells are the mechanisms used by the immune system to maintain tolerance to self-antigens and prevent the development of autoimmunity [21, 22]. Egr $2 / 3$ genes are able to induce anergy in both in vitro and in vivo conditions [23-25]. Egr2 and Egr3 are negative regulators of $\mathrm{T}$ cell activation and inhibit $\mathrm{T}$ cell function by blocking the upregulation of NAB2 and Egr1 [25]. NAB2 by acting as a coactivator collaborates with Egr1 and promotes IL-2 production [26]. Cbl-b was increased in cells overexpressing Egr2 and Egr3, and T cells from Egr $3^{-/-}$had much less Cbl-b in comparison to controls from wild-type mice, supporting the hypothesis that Egr2 and Egr3 can promote upregulation of inhibitory genes involved in T cell activation [27]. Silencing Egr2 gene expression prevents full induction of anergy and increase CD3+CD28- stimulated cells responsiveness to stimulation, resulting in restored proliferation and IL-2 
production in anergic cells in which it demonstrate Egr2 role in conferring the anergic state [28]. A recent study has identified Ndrg1 as a T cell clonal anergy factor induced by Egr2 that is up-regulated by anergic signaling and maintained at high levels in resting anergic $\mathrm{T}$ cells, leading to inhibition of T-cell mediated inflammation [29].

\section{Egr transcription factors and the development of inflammation and systemic autoimmune disease}

Toxic substances released by the activities of virulence factors, allergens, foreign bodies and necrotic cells are stimulants for inflammatory responses and inflammation initiates a physiological response to deregulated homeostasis due to stimulants. This allows the recruitment of non-residential leukocytes to the local sites of infection or tissue injury [30]. Inflammation is generally divided by two categories in which it may be acute, leading to the resolution of inflammation or chronic with persistent with presence of inflammatory molecules and cells [31]. Egr2 and Egr3 are essential for the control of inflammation and antigen induced proliferation of B and T cells, So analysis of CD2-Egr $2^{-/}$and $\mathrm{Egr}^{-/-}$mice resulted in the reduced activation of SOCS1 and SOCS3 in which led to increased activation of IFN-g and Th17 polarization respectively, suggesting vital role of Egr2 and Egr3 not only in preventing the development of autoimmune disease, but also for limiting immunopathology during productive adaptive immune responses (Fig. 2) [13, 32]. Deficiency in SOCS1 and SOCS3 leads to activation of peripheral T cells, resulting in the development of inflammatory disease [33, 34]. STAT1 and STAT3, the major signal transducers pathways in which control the differentiation and development of Th1 and Th17, are negatively regulated by suppressor of cytokine signaling (SOCS) 1 and SOCS3 respectively [35].

Moreover, Egr2 regulate Batf, a transcriptional factor that regulates IL-17 expression and Th17 differentiation, by blocking it's binding to promoters of Th17 cytokines, thus contributing to prevent immunopathology in autoimmune disease [36].

Zhu et al. [37], showed that in Egr2 cKO, CD44 ${ }^{\text {high }} \mathrm{T}$ cells are hyperactive and hyperproliferative in vivo, which can lead to development of a late onset lupus-like autoimmune disease characterized by hair loss, skin lesions, severe glomerulonephritis, the accumulation of IFN- $\gamma$ and IL-17 producing CD4+ T cells, significant infiltration of T cells into multiple organs and increased total serum Ig in comparison to age matched WT mice. So, this indicated intrinsic role of Egr2 not only in proliferation and inflammation of effector $\mathrm{T}$ cells but also in prevention of developing lupus-like autoimmune disease. CD44 ${ }^{\text {high }}$ is a marker for memory and effector T cells [38]. Significant production of IL-17 and IFN- $\gamma$ is closely related to the pathogenesis of systemic lupus erythematosus [39]. Moreover, one study demonstrated that diminished expression of Egr2 and Egr3 was ameliorated by a peptide, hCDR1, treatment in SLE by downregulation of Akt phosphorylation in which led to

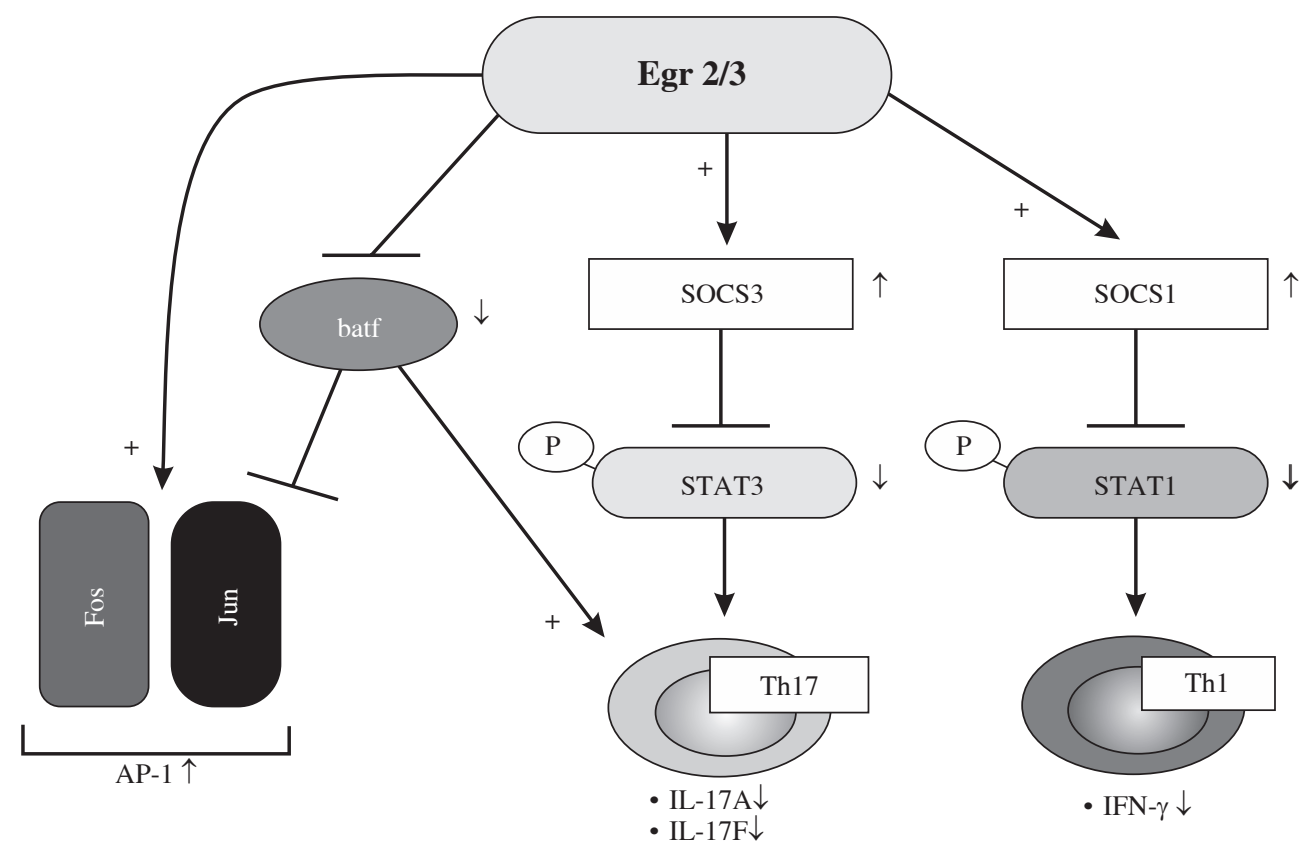

Fig. 2. Overview of the role of Egr2 and Egr3 functions [32] 
down-regulated IL-2 mRNA expression and IFN- $\gamma$ secretion. On the other hand, it was associated with increased expression of E3 ligase Cbl-b and immunosuppressive cytokine TGF $\beta$, inducing anergy. So, it showed negative regulators Egr2 and Egr3 have indispensible role for the maintenance of SLE [40]. Akt is an important kinase that plays a key role in T cell survival, activation and proliferation and it provides costimulatory signals and can activated either by CD28 or following cross-linking of TCR [41].

\section{Concluding remarks and perspectives}

Egr transcription factors are involved in a vast array of functions, particularly their role in regulating of immune response has been investigated. Their role in the induction of differentiation of lymphocyte precursors, B and T cell activation, as well as their involvement in the anergy has been demonstrated.

Egr2 acts in many ways in a manner expected of a master regulator for cell proliferation and death, some having proliferative functions in specific contexts and others promoting growth arrest. These differences can be influenced by the cell types and signaling environment.

Moreover, Egr2 and 3 act as negative regulators of immune response, in specific by their effect in the peripheral tolerance mechanism such as anergy. Furthermore, Egr2 and 3 deficiency has been related to the development of autoimmune diseases in murine conditional Egr2 knockout models.

Also, their role in apoptosis through FasL promoter has been addressed, however this has not been consistent and corroborated. Although TCR signaling seems to be the key elements for the expression of Egr transcriptional factors, little is known about the precise mechanism of Egr expression which needs to be elucidated.

\section{References}

1. Poirier R, Cheval H, Mailhes C, et al. (2008): Distinct functions of Egr gene family members in cognitive processes. Front Neurosci 2: 47-55.

2. Warner LE, Mancias P, Butler IJ, et al. (1998): Mutations in the early growth response 2 (EGR2) gene are associated with hereditary myelinopathies. Nat genet 18: 382-384.

3. Decker EL, Skerka C, Zipfel PF (1998): The Early Growth Response Protein (EGR-1) Regulates Interleukin-2 Transcription by Synergistic Interaction with the Nuclear Factor of Activated T Cells. J Biol Chem 273: 26923-26930.

4. Bettini M, Xi H, Milbrandt J, Kersh GJ (2002): Thymocyte Development in Early Growth Response Gene 1-Deficient Mice. J Immunol 169: 1713-1720.

5. Rivera RR, Johns CP, Quan J, et al. (2000): Thymocyte selection is regulated by the helix-loop-helix inhibitor protein, Id3. Immunity 12: 17-26.

6. Bain G, Cravatt CB, Loomans C, et al. (2001): Regulation of the helix-loop-helix proteins, E2A and Id3, by the Ras-ERK MAPK cascade. Nat Immunol 2: 165-171.
7. Krishnaraju K, Nguyen HQ, Liebermann DA, Hoffman B (1995): The Zinc Finger Transcription Factor Egr-1 Potentiates Macrophage Differentiation of Hematopoietic cells. Mol Cell Biol 15: 5499-5507.

8. O'donovan KJ, Tourtellotte WG, Milbrandt J, Baraban J M (1999): The Egr family of transcription-regulatory factors: Progress at the interface of molecular and systems neuroscience. Trends Neurosci 22: 167-173.

9. Tourtellotte WG, Nagarajan R, Auyeung A, et al. (1999): Infertility associated with incomplete spermatogenic arrest and oligozoospermia in Egr4-deficient mice. Development 126: 5061-5071.

10. Bae CJ, Jeong J, Saint-Jeannet JP (2015): A novel function for Egr4 in posterior hindbrain development. Sci Rep 5: 7750.

11. Li S, Symonds AL, Zhu B, et al. (2011): Early growth response gene-2 (Egr-2) regulates the development of B and T cells. PLoS One 6: e18498.

12. Germain RN (2002): T-cell development and the CD4-CD8 lineage decision. Nat Rev Immunol 2: 309-322

13. Li S, Miao T, Sebastian M, et al. (2012): The transcription factors Egr2 and Egr3 are essential for the control of inflammation and antigen-induced proliferation of $\mathrm{B}$ and $\mathrm{T}$ cells. Immunity 37: 685-696.

14. Lemaire P, Revelant O, Bravo R, Charnay P (1998): Two mouse genes encoding potential transcription factors with identical DNA-binding domains are activated by growth factors in cultured cells. Proc Natl Acad Sci 85: 4691-4695.

15. Unoki M, Nakamura Y (2001): Growth-sppressive effects of BPOZ and EGR2, two genes involved in hte PTEN signaling pathway. Oncogene 20: 4457-4465.

16. Unoki M, Nakamura Y (2003): EGR2 induces apoptosis in various cancer cell lines by direct transactivation of BNIP3L and BAK. Oncogene 22: 2172-2185.

17. Salotti J, Sakchaisri K, Tourtellotte WG, Johnson PF (2015): An Arf-Egr-C/EBP beta pathway linked to ras-induced senescence and cancer. Mol Cell Biol 35: 866-883.

18. Gabet Y, Baniwal SK, Leclerc N, et al. (2010): Krox20/EGR2 deficiency accelerates cell growth and differentiation in the monocytic lineage and decreases bone mass. Blood 116 : 3964-3971.

19. Parkinson DB, Bhaskaran A, Droggiti A, et al. (2004): Krox-20 inhibits Jun-NH2-terminal kinase/c-Jun to control Schwann cell proliferation and death. J Cell Biol 164: 385394.

20. Grunewald TG, Bernard V, Gilardi-Hebenstreit P, et al. 2015. Chimeric EWSR1-FLI1 regulates the Ewing sarcoma susceptibility gene EGR2 via a GGAA microsatellite. Nat Genet 47: 1073-1078.

21. Schwartz RH (2003): T cell Anergy. Annu Rev Immunol 21: 305-334.

22. Hargreaves RG, Borthwick NJ, Gilardini Montani MS, et al. (1997): Dissociation of T cell anergy from apoptosis by blockade of Fas/Apo-1 (CD95) signaling. J Immunol 158: 3099-3107.

23. Zheng Y, Zha Y, Spaapen RM, et al. (2013): Egr2-dependent gene expression profiling and ChIP-Seq reveal novel biologic targets in T cell anergy. Mol immunol 55: 283-291.

24. Zheng Y, Zha Y, Driessens G, et al. (2012): Transcriptional regulator early growth response gene 2 (Egr2) is required for T cell anergy in vitro and in vivo. J Exp Med 209: 2157-2163.

25. Collins S, Lutz MA, Zarek PE, et al. (2008): Opposing regulation of T cell function by Egr-1/NAB2 and Egr-2/Egr-3. Eur J Immunol 38: 528-536. 
26. Collins S, Wolfraim LA, Drake CG, et al. (2006): Cutting Edge: TCR-Induced NAB2 Enhances T Cell Function by Coactivating IL-2 Transcription. J Immunol 177: 8301-8305.

27. Safford M, Collins S, Lutz MA, et al. (2005): Egr-2 and Egr-3 are negative regulators of $\mathrm{T}$ cell activation. Nat Immunol 6: 472-480.

28. Harris JE, Bishop KD, Phillips NE, et al. (2004): Early Growth Response Gene-2, a Zinc-Finger Transcription Factor, Is Required for Full Induction of Clonal Anergy in CD4+ T Cells. J Immunol 173: 7331-7338.

29. Oh Y M, Park HB, Shin JH, et al. (2015): Ndrg1 is a T-cell clonal anergy factor negatively regulated by CD28 costimulation and interleukin-2. Nat Commun 6: 8698.

30. Noah TA, Weil ZM, Nelson RJ (2012): Inflammation: Mechanisms, Costs, and Natural Variation. Annu Rev Ecol Sys 43, 385-406.

31. Ryan GB, Majno G (1997): Acute inflammation: A review. Am J Pathol 86: 183-276.

32. Sumitomo S, Fujio K, Okamura T, Yamamoto K (2013): Egr2 and Egr3 are the unique regulators for systemic autoimmunity. JAKSTAT 2: e23952.

33. Chong MM, Metcalf D, Jamieson E, et al. (2005): Suppressor of cytokine signaling-1 in T cells and macrophages is critical for preventing lethal inflammation. Blood 106: 1668-1675.

34. Croker BA, Metcalf D, Robb L, et al. (2004): SOCS3 Is a Critical Physiological Negative Regulator of G-CSF Signaling and Emergency Granulopoiesis. Immunity 20: 153-165.

35. Ramgolam VS, Markovic-Plese S. (2011): Regulation of suppressors of cytokine signaling as a therapeutic approach in autoimmune diseases, with an emphasis on multiple sclerosis. J Signal Transduct 2011: 635721.

36. Miao T, Raymond M, Bhullar P, et al. (2012): Early growth response gene- 2 controls IL-17 expression and Th17 differentiation by negatively regulating Batf. J Immunol 190: 58-65.

37. Zhu B, Symonds ALJ, Martin JE, et al. (2008): Early growth response gene 2 (Egr-2) controls the self-tolerance of T cells and prevents the development of lupuslike autoimmune disease. J Exp Med 205: 2295-2307

38. Schumann J, Stanko K, Schliesser U, et al. (2015): Differences in CD44 Surface Expression Levels and Function Discriminates IL-17 and IFN-gamma Producing Helper T Cells. PLoS One 10: e0132479.

39. Crispin JC, Oukka M, Bayliss G, et al. (2008): Expanded Double Negative T Cells in Patients with Systemic Lupus Erythematosus Produce IL-17 and Infiltrate the Kidneys. J Immunol 181: 8761-8766.

40. Sela U, Dayan M, Hershkoviz R, et al. (2008): A Peptide That Ameliorates Lupus Up-Regulates the Diminished Expression of Early Growth Response Factors 2 and 3. J Immunol 180: 1584-1591.

41. Kane LP, Weiss A. (2003): The PI-3 Kinase/Akt pathway and T cell activation: Pleiotropic pathways downstream of PIP3. Immunol Rev 12: 7-20. 